\title{
A hydrodynamic mechanism of meteor ablation
}

\section{Approximate analytical solution}

\begin{abstract}
Oleksandr G. Girin
Odesa National Maritime University, Mechnikova str., 34, 65029 Odesa, Ukraine

e-mail: club21@ukr.net

Received 22 August 2016 / Accepted 24 July 2018

ABSTRACT

Aims. We aim to obtain an approximate analytical solution to the equations of meteoroid dynamics within the frames of the suggested mechanism of ablation due to meteoroid melt spraying.

Methods. We have described the droplet breakaway of the melt in terms of hydrodynamic instability theory for the case of a shallow angle of meteoroid entry. The differential equation of meteoroid spraying was derived, together with the equation for the distribution function of sprayed particles by sizes. The latter was obtained considering two different frameworks for meteoroid motion law: empirical and theoretical.

Results. The trinal problem of an ablating meteoroid is solved analytically. The approximate solution is found for the system of equations of motion, ablation and number of sprayed droplets. The latter yields the equation for the distribution function of breakaway droplets by sizes, for which the approximate solution is obtained by providing the intermediate and final number distributions. The meteoroid ablation-deceleration balance remains in the regime of dynamic ablation, when the meteoroid velocity deficit due to aerodynamic drag is negligible until the instant of almost total meteoroid destruction. The responsible " $h$ "-criterion is found, which depends only on the physical properties of the media, so that for melts of materials with lower viscosity such as iron the balance is closer to dynamic ablation than for the stony melt. The differential equation for meteoroid spraying is derived and integrated, showing a near-linear decrease of the meteoroid radius with time.

Conclusions. The proposed spraying model allows us to obtain approximate laws of meteoroid dynamics, which can serve as a base of a comprehensive study of meteor formation and evolution. The model provides intermediate and final number distributions of the breakaway droplets by sizes through direct numerical integration of the governing equations, or, alternatively, in the form of approximate relationships. The theory can be extended to the general case of a molten meteoroid entering atmosphere at an arbitrary angle.
\end{abstract}

Key words. hydrodynamics - instabilities - methods: analytical - methods: numerical - meteorites, meteors, meteoroids

\section{Introduction}

Hydrodynamic mechanism of meteoroid ablation as a quasicontinuous spraying of the meteoroid melt was considered in part one of this work (Girin 2017), which is referred to here as Paper I. The mechanism consists in the meteoroid molten substance spraying in the form of fine liquid droplets due to the hydrodynamic "gradient" instability of conjugated (air-melt) boundary layers at the meteoroid body surface. A shallow-angle trajectory and the regime of outstripping melting are assumed in this paper for a compact, almost completely molten meteoroid, the radius of which lies within a certain range. Under these assumptions, behaviour of the meteoroid is equivalent to the one of a liquid drop in a high velocity uniform air flow, so that the gradient instability theory can be directly applied to the process of the liquid particle generation during the meteoroid flight to determine the particle sizes and the instant of their breakaway. As a result, the approximate analytic solution proposed in this study can also be employed to obtain the distribution function of the sprayed molten droplets. However, this problem is complicated by the coupled processes of meteoroid deceleration and ablation, which are mutually influenced.

A meteoroid of spherical shape is considered, which begins to ablate when moving in the continuum regime. In general, the transiency and complexity of the flow geometry around a spherical meteoroid make it necessary to carry out a numerical treatment of the ablation process. The numerical scheme of calculation of the particle breakaway process was elaborated in Paper I, which takes into account the temporal and spatial variations of hydrodynamic parameters along the meteoroid surface. Variations of both (gas-liquid) boundary layer thicknesses were taken into account by applying basic boundary-layer theory relations. The numerical scheme has allowed us to obtain intermediate and final number distributions of the broken away particles by sizes, as well as the meteoroid mass loss law.

Notwithstanding its importance, the melt spraying represents only the initial process of the aerosol formation in a meteor wake. The meteoroid deceleration, mass loss and ensuing processes of the sprayed particles' acceleration, evaporation, cooling, and solidification results in an involved quantitative description of the overall meteor phenomenon. The processes taking place in meteoric persistent trains set up the physico-chemical conditions in meteor plasmas, which determines afterglow emission spectra and provides very useful data to help understand the composition of the progenitor meteoroids (Madiedo et al. 2014; Drouard et al. 2018). We note that the orders of magnitude of the main parameters of the problem differ significantly one from each other, resulting in difficulties during the calculations. The general problem requires thus simplifications, such as the application of approximate solutions for some elementary processes. 
Such a solution of the differential equations of meteoroid motion and ablation is obtained in the present work under some additional restrictions. This solution makes it possible to derive an equation for the distribution function of all sprayed particles by sizes, and to solve it in the form of approximate expressions. The distribution function is the key object in many of the atmospheric dust investigations.

\section{Equation for broken-away droplet quantification}

The general formulation of this problem was described in Paper I, but we refer to some specific points here. It was noted in Paper I that the instability of the meteoroid melt is generated by the gradient flow in the liquid boundary layer, which is imposed tangentially by the co-flowing air. The fastest unstable disturbance is of prime interest in practical applications since it may result in a molten particle breaking-away at the nonlinear stage of the disturbance development. Amplification of the fastest disturbances at various area elements of the meteoroid surface is governed by the dependencies of the wavenumber, $\Delta_{\mathrm{f}}\left(W e_{\mathrm{s}}\right)$, and the growth rate factor, $\operatorname{Im}\left[\Omega_{\mathrm{f}}\left(W e_{\mathrm{s}}\right)\right]$, on the "surface" Weber number, $W e_{\mathrm{s}}=\rho_{\mathrm{m}} V_{\mathrm{s}}^{2} \delta_{\mathrm{m}} / \Sigma$. When applying these dependencies to meteoroid, we found that there exists a critical point on the meteoroid surface, $\varphi_{\mathrm{cr}}(t)$, where the critical value of the surface Weber number occurs: $W e_{\mathrm{s}}\left(\varphi_{\mathrm{cr}}\right)=W e_{\text {s.cr. }}=3.08$. This critical point divides the meteoroid surface into stable, $\varphi<\varphi_{\mathrm{cr}}$, and unstable, $\varphi>\varphi_{\mathrm{cr}}$, regions. Here $\varphi$ is the polar angle of the area element on the meteoroid surface (Fig. 3 of Paper I). Assuming a potential flow past a spherical meteoroid, $V_{\mathrm{a}}=1.5\left(V_{\infty}-w\right) \sin \varphi$, taking the distribution of the air boundary layer thickness along the spherical surface in Ranger's (1972) form, $\delta_{\mathrm{a}}(\varphi, t)=2.2 R(t) R e_{\infty}^{-0.5}(t) \Psi(\varphi)$, and taking into account Eqs. (3) of Paper I, we obtain the following expressions for the boundary layer thickness in the melt and for the gas-liquid mutual velocity at the interface, respectively:

$\delta_{\mathrm{m}}(\varphi, t)=2.2 \frac{\alpha^{1 / 3}}{\mu^{2 / 3}} \frac{R(t)}{\operatorname{Re}_{\infty}^{0.5}(t)} \Psi(\varphi)$,

and

$V_{\mathrm{s}}(\varphi, t)=\frac{\alpha^{1 / 3} \mu^{1 / 3}}{1+\alpha^{1 / 3} \mu^{1 / 3}} 1.5\left[V_{\infty}-w(t)\right] \sin \varphi$.

Here $\Psi(\varphi) \equiv\left[(6 \varphi-4 \sin 2 \varphi+0.5 \sin 4 \varphi) / \sin ^{5} \varphi\right]^{0.5}, \alpha=$ $\rho_{\mathrm{a}} / \rho_{\mathrm{m}}, \mu=\mu_{\mathrm{a}} / \mu_{\mathrm{m}}$ are the air-to-melt density and viscosity ratios, assuming that melt is the Newtonian fluid. Then, the condition $W e_{\mathrm{s}}(\varphi)>3.08$ for the gradient instability existence at any area element of meteoroid surface can be rewritten as

$\frac{2.475}{\left[1+(\alpha \mu)^{1 / 3}\right]^{2}} \sqrt{\tilde{R}(\tau)[1-W(\tau)]^{3}} \sin ^{2} \varphi \Psi(\varphi) \mathrm{GI} \geq K$.

Here $\tilde{R}=R / R_{0}$ and $W=w / V_{\infty}$ are the dimensionless current meteoroid radius and velocity deficit due to deceleration, $R_{0}$ is the initial meteoroid radius, $\tau=t / t_{\mathrm{ch}}, t_{\mathrm{ch}}=2 R_{0} / \alpha^{0.5} V_{\infty}$ is the timescale, $V_{\infty}$ is the initial meteoroid velocity, $\mathrm{GI} \equiv W e_{\infty} / R e_{\infty}^{0.5}$ the gradient instability criterion and $K$ is a constant. The equality in Eq. (2) at $K=3.08$ determines the value of $\varphi_{\mathrm{cr}}(\tau)$; at GI $>0.4$ and $\tau=0$ (so that $\tilde{R}=1, W=0$ ) we have $\varphi_{\mathrm{cr}}<\pi / 2$. This means that the part of the surface adjacent to meteoroid edge, $\varphi_{\mathrm{cr}}<\varphi<\pi / 2$, is unstable, providing the possibility of melt spraying. The criterion GI is usually large in the meteor phenomenon, GI $\gg \mathrm{GI}_{\mathrm{cr}}=0.4$, so that the values $\varphi_{\mathrm{cr}}$ are small enough: $\varphi_{\text {cr }} \ll \pi$ (e.g. at GI $=43.5$ we have $\varphi_{\text {cr }}=8.8^{\circ}$ ), such that most of the meteoroid surface generates a mist of liquid particles.

The key parameters of the breakaway process, namely, the disturbance wavelength $\lambda_{\mathrm{f}}$, and the characteristic time $t_{\mathrm{f}}$ of its amplitude growth, are both varied along the meteoroid surface and in time. In order to include the breakaway mechanics into the calculating algorithm, we have divided the meteoroid surface on a system of plane elementary areas (more exactly - spherical belts in the axially symmetrical flow) of the width $\Delta l_{i}=R(t) \Delta \varphi_{i}$ (Fig. 4 of Paper I), so that at every time-step the flow parameters may be treated as constant at each ith elementary area. We applied the results of instability analysis to the locally plane flow at each unstable area element, which makes it possible the spraying quantification. This mechanism of instability is governed by the regularities $\Delta_{\mathrm{f}}\left(W e_{\mathrm{s}}\right), \operatorname{Im}\left(\Omega_{\mathrm{f}}\left(W e_{\mathrm{s}}\right)\right)$, shown in Fig. 5 of Paper I.

Thus, we considered each area element $\Delta l$ on the unstable part of the meteoroid surface, $\varphi_{\mathrm{cr}}<\varphi<\pi / 2$, as a source of tiny melt particles (Fig. 4 of Paper I). It is natural to set the size of the breakaway particle as proportional to half-wavelength of the fastest disturbance $\lambda_{\mathrm{f}}$, while the period of its breaking-away, $t_{\mathrm{per}}$, to the time $t_{\mathrm{f}}$ of e-fold growth of the disturbance amplitude at a given $i$ th area element

$r_{i}=k_{\mathrm{r}} \lambda_{\mathrm{f} i}, \quad k_{\mathrm{r}}<0.25 ; \quad t_{\text {per. } i}=k_{\mathrm{t}} t_{\mathrm{f} i}, \quad k_{\mathrm{t}} \approx 1$,

where $\lambda_{\mathrm{f}}=2 \pi \delta_{\mathrm{m}} / \Delta_{\mathrm{f}}, t_{\mathrm{f}}=\operatorname{Im}^{-1}\left(\Omega_{\mathrm{f}}\right) \delta_{\mathrm{m}}(\varphi) / V_{\mathrm{s}}(\varphi)$.

When the part of the induction time of the disturbance growth, $\tau_{\text {ind } i}=\int \mathrm{d} t / t_{\mathrm{per} . i}$, exceeds unity, it is supposed that the separation of the tori of cross-section radius $r_{i}$ and of number $n_{\text {tor } i}$ occurs at that moment from that area element. The quantity of wavelengths, which are confined within $i$ th area element, is equal to $n_{\text {tor } i}\left(\varphi_{i}\right)=\Delta l_{i}\left(\varphi_{i}\right) / \lambda_{\mathrm{f} i}\left(\varphi_{i}\right)$. Due to the axial symmetry of the flow past meteoroid, this is the quantity of tori of radius $R(t) \sin \varphi_{i}$, which have been broken away from the spherical belt corresponding to $\Delta l_{i}$. Assuming that the liquid torus is quickly breaking into droplets of radius $r_{i}$ in the highspeed air stream, and taking the overall volume of tori, $\Delta v_{i}=$ $n_{\text {tor } i} \cdot \Delta v_{\text {tor } i}=\pi k_{\mathrm{r}}^{2} \lambda_{\mathrm{f} i} 2 \pi R \sin \varphi_{i} \cdot \Delta l_{i}$, divided by the single particle volume, $v_{\mathrm{p} i}=4 / 3 \pi r_{i}^{3}$, we obtain the differential equation for the number $\Delta n$ of newborn particles of radius $r(\varphi, \tau)$ :

$\Delta n=\dot{n}^{\prime}(\varphi, \tau) \Delta \varphi \Delta \tau=B_{2} \sqrt{\tilde{R}(\tau)[1-W(\tau)]^{5}} \frac{\sin ^{2} \varphi}{\Psi^{3}(\varphi)} \Delta \varphi \Delta \tau$,

$\tilde{r}(\varphi, \tau)=B_{1} T(\tau) \Psi(\varphi), \quad T=\sqrt{\frac{\tilde{R}(\tau)}{1-W(\tau)}}$,

which have been broken away from the area element $\Delta \varphi$ in the period $\Delta \tau$. Here $\dot{n}^{\prime}(\varphi, \tau)$ is the rate of particle production, in which the coefficients, given by

$B_{1}=\frac{4.4 \pi k_{\mathrm{r}}}{\Delta_{\mathrm{f}} \sqrt{2 R e_{\infty}}} \frac{\alpha^{1 / 3}}{\mu^{2 / 3}}$,

and

$B_{2}=\frac{0.21 \Delta_{\mathrm{f}}^{2} \operatorname{Im}\left(\Omega_{\mathrm{f}}\right) \sqrt{2 R e_{\infty}^{3}}}{\pi k_{\mathrm{r}} k_{\mathrm{t}}\left[1+(\alpha \mu)^{1 / 3}\right]} \frac{\mu^{7 / 3}}{\alpha^{7 / 6}}$,

can be interpreted as scaling parameters for the particle sizes and number, respectively (Girin 2011a), and the superscript dot stands for the differentiation with respect to $\tau$, while ' - to $\varphi$; $\tilde{r}=r / R_{0}$. 


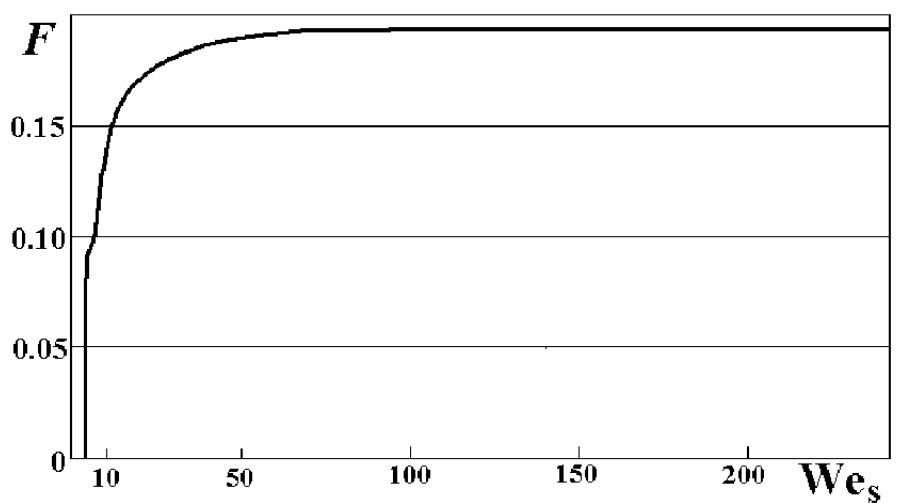

Fig. 1. Dimensionless function $F \equiv \operatorname{Im}\left(\Omega_{\mathrm{f}}\right) / \Delta_{\mathrm{f}}$ versus "surface" Weber number $W e_{\mathrm{s}}=\rho_{\mathrm{m}} V_{\mathrm{s}}^{2} \delta_{\mathrm{m}} / \Sigma$.

\section{Equation of meteoroid mass losses}

The mechanism of melt spraying described in Sect. 2 predetermines an alternative form of differential equation of meteoroid ablation, which differs from the classic equation of the general physical theory of meteors (Bronshten 1983). Since the elementary breakaway process occurs in a time period $t_{\text {per }}(\varphi)=$ $k_{\mathrm{t}} t_{\mathrm{f}}(\varphi)=k_{\mathrm{t}} \delta_{\mathrm{m}}(\varphi) / V_{\mathrm{s}}(\varphi) \operatorname{Im}\left(\Omega_{\mathrm{f}}\right)$, the mass efflux rate can be expressed as

$$
\begin{aligned}
& \frac{\Delta m}{\Delta t}(\varphi, t)=\frac{\rho_{\mathrm{m}} \Delta v(\varphi, t)}{t_{\mathrm{per}}(\varphi, t)} \\
& =\frac{\rho_{\mathrm{m}} 2 \pi^{2} k_{\mathrm{r}}^{2} R(t) \lambda_{\mathrm{f}}(\varphi, t) \sin \varphi V_{\mathrm{s}}(\varphi, t) \operatorname{Im}\left[\Omega_{\mathrm{f}}\left(W e_{\mathrm{s}}(\varphi, t)\right)\right]}{k_{\mathrm{t}} \delta_{\mathrm{m}}(\varphi, t)} \Delta l(\varphi, t) .
\end{aligned}
$$

By substituting $\lambda_{\mathrm{f}}=2 \pi \delta_{\mathrm{m}} / \Delta_{\mathrm{f}}$ in Eq. (6), taking into account $\Delta l=R(t) \Delta \varphi$, and by integrating over the windward surface from $\varphi=\varphi_{\mathrm{cr}}(t)$ to the meteoroid edge $\varphi=\pi / 2$, we obtained the rate of meteoroid mass diminishing

$$
\begin{aligned}
& \frac{\mathrm{d} m}{\mathrm{~d} t} \\
& \quad=-\frac{4 \pi^{3} k_{\mathrm{r}}^{2}}{k_{\mathrm{t}}} \rho_{\mathrm{m}} R^{2}(t) \int_{\varphi_{\mathrm{cr}}(t)}^{\pi / 2} \frac{V_{\mathrm{s}}(\varphi, t) \operatorname{Im}\left[\Omega_{\mathrm{f}}\left(W e_{\mathrm{s}}(\varphi, t)\right)\right] \sin \varphi}{\Delta_{\mathrm{f}}\left[W e_{\mathrm{S}}(\varphi, t)\right]} \mathrm{d} \varphi .
\end{aligned}
$$

To simplify the integrand, we note that the function $F\left(W e_{\mathrm{s}}\right) \equiv \operatorname{Im}\left[\Omega_{\mathrm{f}}\left(W e_{\mathrm{s}}\right)\right] / \Delta_{\mathrm{f}}\left(W e_{\mathrm{s}}\right)$, which in Eq. (7) defines an influence of the fastest disturbance parameters on the mass efflux rate, is almost constant for $W e_{\mathrm{s}} \gg W e_{\text {s.cr. }}=3.08$ (Fig. 1). It sharply increases in the vicinity of the critical point, so that we can neglect the mass efflux rate at $\mathrm{We}_{\mathrm{s} . \mathrm{cr} .}<\mathrm{We}_{\mathrm{s}}<W e_{\mathrm{s}}\left(\varphi_{\mathrm{lt}}\right) \approx$ 5.0. Besides, this vicinity must be avoided, since the fastest unstable wave length is greater here than the meteoroid diameter, $\lambda_{\mathrm{f}}>2 R(t)$; as well, its characteristic time is greater than the meteoroid breakup time $t_{\text {spr }}$ (see Fig. 5 of Paper I). Therefore, the corresponding unstable disturbances are not able to be accomplished until the overall breakup time. Consequently, the lower limit in Eq. (7) must be replaced by such a value $\varphi_{\text {lt }}>\varphi_{\text {cr }}$, for which $k_{\mathrm{r}} \lambda_{\mathrm{f}}\left(\varphi_{\mathrm{lt}}, t\right) \lesssim R(t), \quad k_{\mathrm{t}} t_{\mathrm{f}}\left(\varphi_{\mathrm{lt}}\right) \lesssim t_{\mathrm{spr}}$. Taking this into account, we were able to adopt $F\left(W e_{\mathrm{s}}\right)=0.18$ as the average constant value within the spraying interval $\varphi_{\mathrm{lt}}(t)<\varphi \leq \pi / 2$. This assumption is valid for sufficiently large values of the gradient instability criterion: GI $\gg \mathrm{GI}_{\mathrm{cr}}=0.4$, when $\varphi_{\mathrm{cr}}, \varphi_{\mathrm{lt}} \ll \pi$, so that the inequality $W e_{\mathrm{s}} \gg \mathrm{We}_{\mathrm{s} . c r}=3.08$ is satisfied at the majority of the meteoroid surface. Then from Eq. (7) we will have

$$
\begin{aligned}
\frac{\mathrm{d} m}{\mathrm{~d} t}= & -0.18 \frac{6 \pi^{3} k_{\mathrm{r}}^{2} \rho_{\mathrm{m}}}{k_{\mathrm{t}}\left[1+(\alpha \mu)^{1 / 3}\right]}(\alpha \mu)^{1 / 3} R^{2}(t)\left[V_{\infty}-w(t)\right] \\
& \times \int_{\varphi \mathrm{lt}(t)}^{\pi / 2} \sin ^{2} \varphi \mathrm{d} \varphi,
\end{aligned}
$$

with the help of Eqs. (2). Calculating the integral, we obtain the kinetic equation of meteoroid mass loss:

$$
\begin{aligned}
\frac{\mathrm{d} m}{\mathrm{~d} t}= & -\frac{1.08 \pi^{3} k_{\mathrm{r}}^{2} \rho_{\mathrm{m}}}{k_{\mathrm{t}}\left[1+(\alpha \mu)^{1 / 3}\right]}(\alpha \mu)^{1 / 3} R^{2}(t)\left[V_{\infty}-w(t)\right] \\
& \times\left(\frac{\pi}{4}-\frac{\varphi_{\mathrm{lt}}(t)}{2}+\frac{\sin 2 \varphi_{\mathrm{lt}}(t)}{4}\right) .
\end{aligned}
$$

The right-hand side of Eq. (9) expresses the dependence of mass efflux rate on all the main parameters of the process: current meteoroid radius, relative air-meteoroid velocity, physical properties of the media. Surface tension $\Sigma$ has a stabilizing effect implicitly via the parameter $\varphi_{1 \mathrm{lt}}$ : as $\Sigma$ increases, values of $W e_{\mathrm{s}}$ decrease at each area element of the meteoroid surface, so that the critical point ascends to the meteoroid edge, increasing $\varphi_{1 \mathrm{t}}$ and decreasing the spraying surface area as a consequence. Equation (9) may be rewritten in the dimensionless form:

$$
\frac{\mathrm{d} M}{\mathrm{~d} \tau}=-A \tilde{R}^{2}(\tau)[1-W(\tau)]\left(1-\frac{2 \varphi_{\mathrm{lt}}(\tau)}{\pi}+\frac{\sin 2 \varphi_{\mathrm{lt}}(\tau)}{\pi}\right),
$$

where $A \equiv \frac{0.405 \pi^{3} k_{\mathrm{r}}^{2}}{k_{\mathrm{t}}\left[1+(\alpha \mu)^{1 / 3}\right]}\left(\frac{\mu^{2}}{\alpha}\right)^{1 / 6}$ has the sense of the characteristic (initial) rate of the mass efflux, $M=m / m_{0}$.

The integration of Eq. (10) to determine meteoroid ablation law, $M(\tau)$, requires the integration of the equation of the meteoroid motion (in order to have $W(\tau)$ known) and Eq. (2) (at $K=5.0)-$ to determine $\varphi_{1 t}(\tau)$. Their intricacy compels us to solve this system equations considering some further simplifying assumptions. We outline the problem solution for the considered case of speedy flows, when GI $\gg \mathrm{GI}_{\mathrm{cr}}=0.4$. The latter subsequently results, with the aid of Eq. (2): $\varphi_{\mathrm{lt}} \ll \pi$, $\sin 2 \varphi_{\mathrm{lt}} \approx 2 \varphi_{\mathrm{lt}}$. Therefore, taking into account the meteoroid spherical shape (i.e. $M=\tilde{R}^{3}$ ) and the identity $W \equiv \alpha^{0.5} \mathrm{~d} X_{\mathrm{M}} / \mathrm{d} \tau$ for the velocity deficit, the integration of Eq. (10) yields:

$$
\begin{aligned}
& M(\tau)=\left[1-\frac{A}{3}\left(\tau-\alpha^{0.5} X_{\mathrm{M}}(\tau)\right)\right]^{3}, \\
& \tilde{R}(\tau)=1-\frac{A}{3}\left(\tau-\alpha^{0.5} X_{\mathrm{M}}(\tau)\right),
\end{aligned}
$$

where $x_{\mathrm{M}}$ is meteoroid displacement due to the aerodynamic drag, $X_{\mathrm{M}}=x_{\mathrm{M}} / 2 R_{0}$. Equations (11) indicate clearly the direct dependence of meteoroid ablation law on its deceleration law, $X_{\mathrm{M}}=X_{\mathrm{M}}(\tau)$. It is much simpler to obtain first the latter law, which can be done in two ways, empirical and theoretical, as was done for a drop in Girin (2011a).

\subsection{Empirical approximation of meteoroid deceleration law}

First, we used the experimental data of Reinecke \& Waldman (1975), which are valid for a liquid drop in the rarefied air conditions relevant for the reentry flight. These data allow us 
to express the law of a liquid sphere motion in the form: $\alpha^{0.5} X_{\mathrm{M}}(\tau)=\tau-[1-\exp (-H \tau)] / H$, where $H=2 \alpha^{0.5}$ has the sense of the characteristic deceleration due to aerodynamic drag. From here $W=1-\exp (-H \tau)$. Taking into account the expression for the dimensionless velocity, $W=\alpha^{0.5} \mathrm{~d} X_{\mathrm{M}} / \mathrm{d} \tau$, Eq. (11) yields the meteoroid ablation law:

$M=\tilde{R}^{3}=[1-h(1-\exp (-H \tau))]^{3}$.

The rate ratio $h=A / 3 H$ reflects the influence of two competing factors, which govern the meteoroid dynamics: the mass reduction rate $(\approx A$, see Eq. (10)) due to spraying, and the rate of relaxational reducing of the air-meteoroid relative velocity $(\approx H)$, which in turn diminishes the mass efflux. Due to the extremely small values of the density ratio $\alpha$ in high atmosphere, values of $H$ are small so that the $h$ values are large for meteoroid: $h>10^{2}$. Equation (12) shows that the meteoroid is completely sprayed at the time instant $\tau_{\mathrm{spr}}=H^{-1} \ln [h /(h-1)]$, which, at $h \gg 1$, approximately equals to $\tau_{\mathrm{spr}}=[H(h-1)]^{-1} \approx 3 / A$. Comparison of the ablation law (12) with experimental data of Reinecke \& Waldman (1975) for atomizing drops indicated their good agreement (Girin 2011a).

\subsection{Theoretical law of ablating meteoroid deceleration}

The second way to obtain the deceleration law of an ablating meteoroid consists of the direct integration of the differential equation of the meteoroid motion:

$\rho_{\mathrm{m}} \frac{4}{3} \pi R^{3}(t) \frac{\mathrm{d} w}{\mathrm{~d} t}=\Gamma \pi R^{2}(t) \rho_{\mathrm{a}}\left[V_{\infty}-w(t)\right]^{2}$,

where $\Gamma$ is the aerodynamic drag coefficient, which for a meteoroid has a value of the order unity (Bronshten 1983). Though the first integral of the system of meteoroid governing equations of the physical theory of meteors can be derived (Pecina \& Koten 2009), the integration is usually carried out either with the assumption that meteor deceleration can be neglected, or with the application of numerical methods.

Next, we show that the dynamic equations of the spraying meteoroid theory (Eq. (14a)) can be integrated analytically. Modifying Eq. (10) in terms of the meteoroid radius, $\tilde{R}=M^{1 / 3}$, we obtained for $\mathrm{GI} \gg \mathrm{GI}_{\mathrm{cr}}$ the coupled differential equations for the interrelated processes of meteoroid ablation and deceleration with respect to the dimensionless variables $\tilde{R}$ and $W$ :

$$
\begin{aligned}
& \frac{\mathrm{d} W}{\mathrm{~d} \tau}=C \frac{(1-W)^{2}}{\tilde{R}} \\
& \frac{\mathrm{d} M^{1 / 3}}{\mathrm{~d} \tau}=\frac{\mathrm{d} \tilde{R}}{\mathrm{~d} \tau}=-\frac{A}{3}(1-W),
\end{aligned}
$$

with the initial conditions $\tilde{R}(0)=1, W(0)=0$; here $C=1.5 \alpha^{0.5} \Gamma$ has the sense of the characteristic (initial) meteoroid deceleration. By eliminating the velocity term from Eqs. (14a) and (14b) we obtained a second-order equation with respect to the meteoroid radius:

$\tilde{R} \frac{\mathrm{d}^{2} \tilde{R}}{\mathrm{~d} \tau^{2}}=\left(\frac{\mathrm{d} \tilde{R}}{\mathrm{~d} \tau}\right)^{2}$.

Here, we have the meteoroid dynamic criterion in the form $h=A / 3 C$, which assumes large values since $C$ is small. At $h>1$ the integration of Eq. (15) with initial conditions $\tilde{R}(0)=1$,
$\dot{\tilde{R}}(0)=-A / 3$ (the latter follows from Eq. (14b)) leads to a solution expressed in terms of power functions. Eventually, we obtain the ablation and deceleration laws in the form

$$
\begin{aligned}
& \tilde{R}=[1-C(h-1) \tau]^{h /(h-1)}, \\
& W=1-[1-C(h-1) \tau]^{1 /(h-1)} .
\end{aligned}
$$

The laws (Eq. (16a)) are applicable until the moment $\tau_{\text {spr }}$, when the meteoroid is totally sprayed, $R=0$. From Eq. (16a) we have: $\tau_{\mathrm{spr}}=[(h-1) C]^{-1}(\approx 3 / A$ for $h \gg 1)$; the same as in Sect. 3.1. The expression for $\tilde{R}$ in Eq. (16a) shows that, due to large $h$ values, the dependence of the ablating meteoroid radius on time is close to linear in the count-down timescale, $\tau_{\text {spr }}-\tau$. This conclusion agrees well with both, the empirically approximated dependence (Eq. (12)), taking into account small values of $H$, and the results from our numerical model (see Paper I). Equation (16b) shows that the spraying proceeds at almost unchangeable air-meteoroid relative velocity. The latter is due to the fact that the characteristic time of meteoroid spraying, $3 / A$, is several orders smaller than the one of deceleration, $C^{-1}$, and the rate ratio of these processes, $h$, is large. However, the meteoroid decelerates suddenly to zero relative velocity at the moment when the meteoroid is completely sprayed, $\tilde{R}=0$. The meteoroid deceleration tends to infinity at that moment; this is caused by the meteoroid radius vanishing, which raises the aerodynamic drag force, as shown by Eq. (14a) at $\tilde{R} \rightarrow 0$. This explains the sharp-ending deceleration of meteoroid, and agrees with previous observations (Bronshten 1983; Simonenko 1974).

From Eqs. (16a) and (16b) we can conclude that the rate ratio $h$ is a dynamic similarity criterion, which determines the form of the main dynamic laws of meteoroid behaviour. Moreover, its value determines the dominant process in the ablationdeceleration competition, which is illustrated by the analysis of the dynamic $(R, U)$-diagrams discussed below.

The obtained laws (Eq. (16a)) show that the overall meteoroid behaviour is regulated by two characteristic parameters: its deceleration $C$ and the mass loss rate $A$. Their values reflect two aspects of the aerodynamic action on meteoroid surface: the integral normal force together with viscous friction force decelerate the meteoroid, while the high-speed tangential slipping along the meteoroid surface causes its instability, which pulls out liquid particles from the melt. The meteoroid reaction is reflected in the character changing of two main dependant parameters its relative velocity $U=1-W$ and its degree of fragmentation, which can be measured by the current dimensionless radius, $\tilde{R}=R / R_{0}$. Thus, the meteoroid reaction can be represented by its portrayal in $(\tilde{R}, U)$-plane, which, depending on $A$ and $C$, forms a set of ballistic $(\tilde{R}, U)$-diagrams showing a variety of ablating meteoroid behaviours in the atmosphere. However, in view of Eq. (16a) the meteoroid $(\tilde{R}, U)$-diagrams are all expressed by the one-parameter set of curves: $U=\tilde{R}^{1 / h}$, with the unique parameter $h$. Examples are shown in Fig. 2, calculated for the meteoroids in the present paper.

The meteoroid flight is exceptionally characterized by small $C$ values, while $A$ has the order of unity, which yields large $h=A / 3 C$. Since the values of $A$ and $C$ are determined mostly by the physical properties of meteoroid substance, for every pure substance there exists a single regime of an ablating meteoroid behaviour, which is independent of the initial meteoroid velocity and size. As the curves in Fig. 2 show, the majority of the ablating meteoroid flight is performed at conditions of an intense air motion past the meteoroid, when the relative air-meteoroid velocity remains close to the initial value $U_{0}=1$. Thus, the 


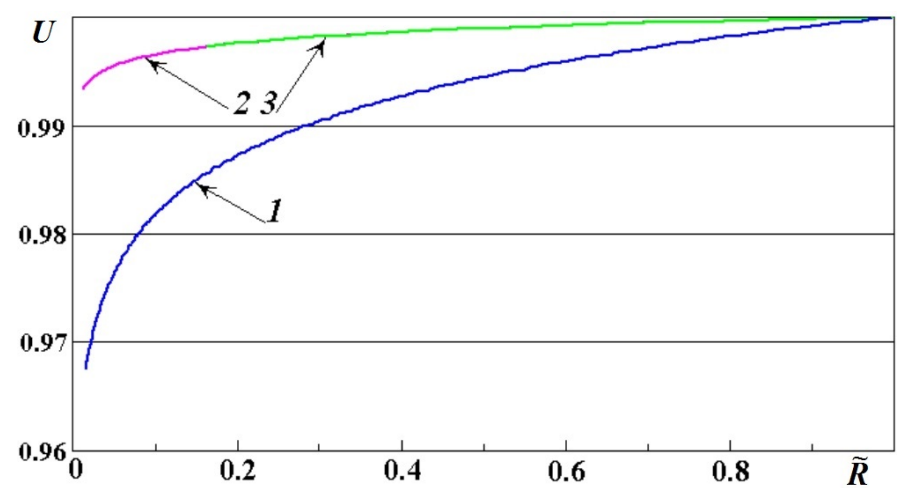

Fig. 2. Dynamic radius-velocity $(\tilde{R}, U)$-diagrams for the stone, $h=125$, (curve $1-V_{\infty}=60 \mathrm{~km} \mathrm{sec}^{-1}$ ) and iron, $h=662$, (crimson + light-green curve $2-V_{\infty}=25 \mathrm{~km} \mathrm{sec}^{-1}$, light-green curve $\mathbf{3}-V_{\infty}=60 \mathrm{~km} \mathrm{sec}^{-1}$ ) meteoroids.

diagrams show that due to large $h$ values the meteoroid is always held in regime of dynamic ablation, when the airflow impact is shifted towards the spraying action, so that the majority of the airstream energy is spent to increase the melt instability, while the minor part is used for meteoroid deceleration. In this connection, one can see in Fig. 2 that iron meteoroid spraying is more efficient than that of stone. For instance, at the same $1 \%$ degree of meteoroid deceleration (or $U=0.99$ ), the degree of the stony meteoroid fragmentation is $\approx 30 \%$ less than that of the iron meteoroid, independently of the initial meteoroid velocity. The effect is expressed also by the shorter amount of time needed for full meteoroid fragmentation and finer pulverization of the iron meteoroid. The reason for this effect lies obviously in the media physical properties (namely, the 30 -fold viscosity difference leads to a difference of approximately 5.3 in $h$ ), as seen in Paper I. When the viscosity of the molten substance is even greater than the stony one, the $(\tilde{R}, U)$-diagram descends lower, showing the possibility of incomplete spraying of completely molten meteoroids. Thus, the parameter $h$ plays a role of a similarity criterion for the dynamic behaviour of meteoroid. Under the adopted assumptions for the considered class of meteoroids, we can conclude that the meteoroids made of the same substance have a similar behaviour during their flight, irrespective of their initial velocities and sizes.

\section{Sprayed-droplet distribution function}

The melt-spraying model allows quantifying the dispersion process in detail. Integrating Eq. (4) for the newborn particle number throughout the domain of spraying in the $(\varphi, \tau)$-plane, we would find the total number of the broken-away particles. However, the most important parameter used in the description of the meteoroid ablation is the transient distribution function of the broken-away particles by sizes, $f_{\mathrm{n}}(\tilde{r}, \tau)=\Delta n(\tilde{r}, \tau) / \Delta \tilde{r}$, which is needed for the quantitative description of the aerosol formation in the meteor wake and its further evolution. Such distributions for ablating meteoroids are unknown to us, neither theoretical, nor empirical. Obtained regularities (Eqs. (4), (5), (12), and (16a)) allow us to know the approximate distribution function of newborn particles by sizes.

In order to obtain the differential distribution function $f_{\mathrm{n}}(\tilde{r}, \tau)$, we need to integrate the equation for particle production rate $\dot{n}^{\prime}(\varphi, \tau)$ (Eq. (4)) along each line $\tilde{r}(\varphi, \tau)=L$ (referred to in the sense of Eq. (5)) over a strip of width $\Delta \tilde{r}=Q$, where

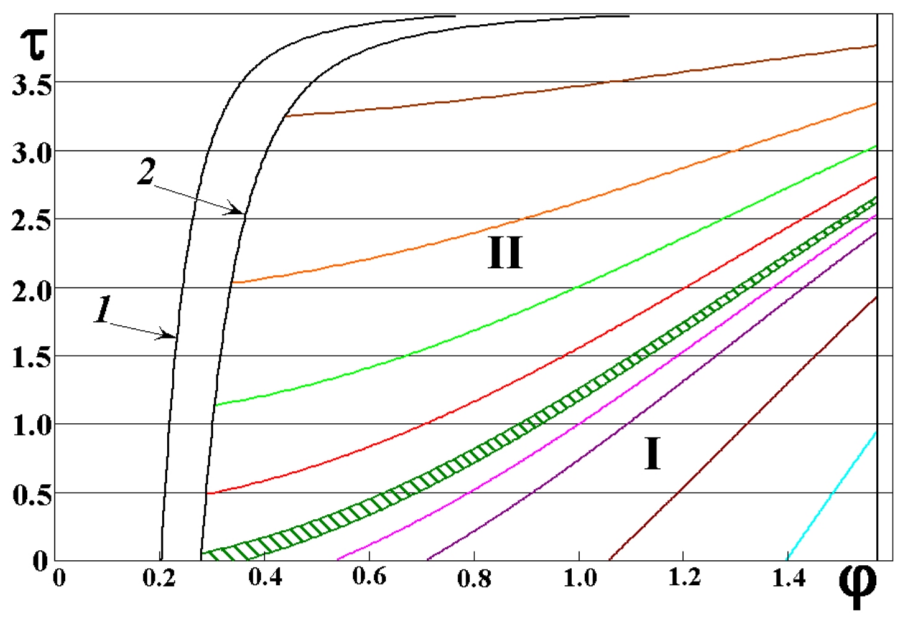

Fig. 3. Set of lines $\tilde{r}(\varphi, \tau)=L$ (coloured) on the plane of events for the iron meteoroid spraying; $\mathrm{GI}=16.8, \tau_{\mathrm{spr}}=4.06$. Black line $\boldsymbol{I}-$ left boundary $\varphi=\varphi_{\mathrm{cr}}(\tau)$ of unstable part of meteoroid surface; black line 2 - left boundary $\varphi=\varphi_{\mathrm{lt}}(\tau)$ of spraying domain. Dashed green band shows the strip of integration, $\Delta \tilde{r}=Q$, for $\tilde{r}_{\mathrm{fix}}=\tilde{r}\left(\varphi_{\mathrm{lt} 0}\right)$

$L$ and $Q$ are constants. Hence, we must first build the spraying domain in the plane of events $(\varphi, \tau)$, which is shown in Fig. 3 together with the calculated set of lines $\tilde{r}(\varphi, \tau)=L=\tilde{r}_{\text {fix }}$. The top and left regions of the spraying domain are bounded by the curve $\varphi=\varphi_{\mathrm{lt}}(\tau)$, while the bottom region by the initial moment $\tau=0$. The meteoroid edge position, $\varphi_{\mathrm{rt}}=\pi / 2$, defines the right boundary of the domain. The spraying domain can be divided conditionally into the two parts, I and II, by the line $\tilde{r}=\tilde{r}_{\text {fix.lt. }}=\tilde{r}\left[\varphi_{\text {lt }}(0), 0\right]$, which leaves the point $\varphi_{\text {lt } 0}=\varphi_{\text {lt }}(0)$. These lines, which lie at the bottom section of region (subdomain I), start at $\tau=0$ and end at $\varphi=\pi / 2$; the lines above, in sub-domain II, start at the left-boundary curve, $\varphi=\varphi_{\mathrm{lt}}(\tau)$, and end at $\varphi=\pi / 2$. It follows then from Eq. (5) that $\tilde{r}(\varphi, \tau)>\tilde{r}_{\text {fix.lt. }}$

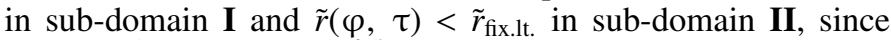
$T(\tau)=[\tilde{R}(\tau) /(1-W(\tau))]^{0.5}$ is the decreasing function (for $h>1$ ) and $\Psi(\varphi)$ is the increasing one.

Spraying starts at $\tau=0$ from the area elements $\Delta \varphi$ within the interval $\varphi_{\text {lt } 0} \leq \varphi \leq \pi / 2$, which represents the unstable part of the meteoroid surface. As Fig. 3 shows, at the very beginning, the breakaway particle sizes lie within the basic range, $\tilde{r}_{\text {fix.lt. }} \leq \tilde{r} \leq \tilde{r}_{\text {fix.rt. }}$, which corresponds to the endpoints of the initial segment $\varphi_{\mathrm{lt} 0} \leq \varphi \leq \pi / 2$. Since $T(0)=1$, here we have $\tilde{r}_{\text {fix.lt. }}=B_{1} \Psi\left(\varphi_{\mathrm{lt} 0}\right)=\sqrt{3.2} B_{1}$ and $\tilde{r}_{\text {fix.rt. }}=B_{1} \Psi(\pi / 2)=\sqrt{3 \pi} B_{1}$. The spraying process then goes gradually into sub-domain II, where $\tilde{r}<\tilde{r}_{\text {fix.lt. }}$, therefore the particle coarse fraction is generated always at the beginning, while the fine fraction - at the end of the ablation process. For this reason, the newborn particle sizes diminish in time, as shown in calculations in Paper I, and clearly seen in the supplemental material of Paper I (video files Movie 1 and Movie 2). The instant when the left boundary of the spraying domain (black line 2) intersects the right boundary is the moment of the meteoroid spraying termination, $\tau=\tau_{\mathrm{spr}}$.

In order to determine $f_{\mathrm{n}}(\tilde{r}, \tau)$, we need to eliminate $\Psi(\varphi)$ from Eq. (4) with the help of Eq. (5) and substitute $\Delta \tau=$ $\Delta \tilde{r} /\left[B_{1} \dot{T}(\tau) \Psi(\varphi)\right]$, which represents the strip with $\Delta \tilde{r}$ width in $\tau$-direction. Then, integrating Eq. (4) along the line $\tilde{r}(\varphi, \tau)=\tilde{r}_{\text {fix }}$ from the lower limit $\left[\varphi_{*}=\varphi\left(0 ; \tilde{r}_{\text {fix }}\right)\right.$ in sub-domain $\mathbf{I}$, and $\varphi_{*}=\varphi_{\mathrm{lt}}\left(\tau ; \tilde{r}_{\mathrm{fix}}\right)$ in sub-domain II] to the upper limit, $\varphi^{*}=\pi / 2$, 
we obtain equation for the distribution function $f_{\mathrm{n}}\left(\tilde{r}_{\mathrm{fix}}, \tau\right)$ :

$$
\begin{aligned}
\Delta n= & f_{\mathrm{n}}\left(\tilde{r}_{\mathrm{fix}}, \tau\right) \Delta \tilde{r}=\frac{2 B_{1}^{3} B_{2}}{(h-1) H \tilde{r}_{\text {fix }}^{4}} \\
& \times \int_{\varphi_{*}}^{\pi / 2} \tilde{R}^{3}[\tau(\varphi)][1-W(\tau(\varphi))] \sin ^{2} \varphi \mathrm{d} \varphi \Delta \tilde{r},
\end{aligned}
$$

in which the dependence $\tau(\varphi)$ ought to be determined from the Eq. (5) for every fixed $\tilde{r}_{\text {fix }}$. Equation (17) clearly shows that both the meteoroid ablation and deceleration laws influence the distribution function.

The evaluation of the curvilinear integral in (17) is a knotty problem in view of kind of the dependence $\tau\left(\varphi ; \tilde{r}_{\text {fix }}\right)$ (see Eq. (5) at $\tilde{r}=\tilde{r}_{\text {fix }}$, and Eqs. (12) and (16a)) so that approximation methods can be useful. We can point out two of the possible ways based on the empirical and analytical approaches of Sect. 2.

\subsection{Distribution function for the empirical approximation}

Using the expressions for the empirical approximation of the meteoroid deceleration law (Eq. (12)), the equation of line $\tilde{r}=$ $\tilde{r}_{\text {fix }}$ in the $(\varphi, \tau)$-plane can be implicitly written as follows:

$\tau\left(\varphi ; \tilde{r}_{\text {fix }}\right)=H^{-1} \ln \left[\frac{h-\left[\tilde{r}_{\text {fix }} / B_{1} \Psi(\varphi)\right]^{2}}{h-1}\right]$,

and Eq. (17) for the distribution function becomes:

$$
\begin{aligned}
\Delta n= & f_{\mathrm{n}} \Delta \tilde{r}=\frac{2 B_{1}^{3} B_{2}}{(h-1)} H \tilde{r}_{\text {fix }}^{4} \\
& \times \int_{\varphi_{*}}^{\pi / 2}[1-h+h \exp (-H \tau)]^{3} \exp (-H \tau) \sin ^{2} \varphi \mathrm{d} \varphi \Delta \tilde{r} .
\end{aligned}
$$

We see from Fig. 3 that in the case $h \gg 1$ the lines $\tilde{r}(\varphi, \tau)=\tilde{r}_{\text {fix }}$ are only slightly curved so that the path of integration in Eq. (19) can be approximated by the straight line $\tau-\tau_{*}=\left(\varphi-\varphi_{*}\right) / a_{\text {ef }}$ with some effective slope $a_{\mathrm{ef}}\left(\tilde{r}_{\mathrm{fix}}, h\right)$. Then, using the tabular integral $\int e^{q \varphi} \sin ^{2} \varphi \mathrm{d} \varphi=e^{q \varphi} / 2 q-$ $e^{q \varphi}(0.5 q \cos 2 \varphi+\sin 2 \varphi) /\left(q^{2}+4\right) \quad$ (Gradshtein \& Ryzhyk 1980) and the laws (12), we obtain, after integrating Eq. (19):

$$
\begin{aligned}
\Delta n\left(\tilde{r}_{\mathrm{fix}}\right) & =f_{\mathrm{n}}\left(\tilde{r}_{\mathrm{fix}}\right) \Delta \tilde{r} \\
& =\frac{B_{1}^{3} B_{2}}{(h-1) \tilde{r}_{\mathrm{fix}}^{4}} \frac{a_{\mathrm{ef}}(\tilde{r})}{H^{2}} \sum_{i=1}^{4} A_{i}\left[\Phi_{i *}\left(\tilde{r}_{\mathrm{fix}}\right)-\Phi_{i}^{*}\left(\tilde{r}_{\mathrm{fix}}\right)\right] \Delta \tilde{r}
\end{aligned}
$$

where $\Phi_{i}\left(\tilde{r}_{\mathrm{fix}}\right)=D^{i}\left(\tilde{r}_{\mathrm{fix}}\right)\left[\sin ^{2} \varphi\left(\tilde{r}_{\mathrm{fix}}\right)+\sin ^{2}\left[\varphi\left(\tilde{r}_{\mathrm{fix}}\right)+\theta_{i}\left(\tilde{r}_{\mathrm{fix}}\right)\right]\right]$ must be calculated at the lower, $\varphi=\varphi_{*}\left(\tilde{r}_{\text {fix }}\right)$, and upper, $\varphi=\varphi^{*}\left(\tilde{r}_{\text {fix }}\right)$, limits of integration, correspondingly; $D\left(\tilde{r}_{\text {fix }}\right)=$ $(h-1) /\left[h-\left[\tilde{r}_{\mathrm{fix}} /\left(B_{1} \Psi(\varphi)\right)\right]^{2}\right] ; A_{i}=0.25 C_{4}^{(i)} h^{i-1}(1-h)^{4-i} ; C_{4}^{(i)}$ are the binomial coefficients, $\theta_{i}=\arcsin \left[1+\left(i H / 2 a_{\mathrm{ef}}\right)^{2}\right]^{-0.5}$ at $h>1$. Values of $\varphi_{*}\left(\tilde{r}_{\text {fix }}\right)$ in sub-domain $\mathbf{I}$ are to be found from Eq. (18) at $\tau=0$. In sub-domain II the system of Eq. (18) of the line $\tilde{r}(\varphi, \tau)=\tilde{r}_{\mathrm{fix}}$ coupled with the equation of the left boundary $\varphi_{\mathrm{lt}}(\tau)$ of the spraying domain (Eq. (2) at $K=5.0$ ) must be solved. In order to simplify the calculations, the expression Eq. (20) is simplified at the upper limit, $\varphi^{*}=\pi / 2$, where $\sin \varphi^{*}=1, \cos \varphi^{*}=0$ and at the lower limit in the initial segment, where $\tau_{*}=0, D=1$.
Analysis of the behaviour of lines $\tilde{r}(\varphi, \tau)=\tilde{r}_{\text {fix }}$ allows us to find the expression for $a_{\text {ef }}$, which is valid for a wide range of $h$ values (Girin 2011b). The effective slope $a_{\text {ef }}$ must be fitted accounting for the influence of both, the initial value $a_{*}=$ $\mathrm{d} \varphi /\left.\mathrm{d} \tau\right|_{\varphi=\varphi_{*}}$ and the mean value $a_{\text {m.v. }}=\left(\varphi^{*}-\varphi_{*}\right) /\left(\tau^{*}-\tau_{*}\right)$. Eventually, the following expressions for $a_{\mathrm{ef}}$ are obtained in sub-domains I and II:

$$
\begin{aligned}
& a_{\mathrm{ef} \mathbf{I}}=\frac{\left(h+2.1 / h^{0.5}\right) a_{\mathrm{m} . \mathrm{v} .} a_{*}}{h a_{*}+a_{\mathrm{m} . v .} h^{-2}+\left(a_{\mathrm{m} . \mathrm{v} .}+a_{*}\right) / h^{0.5}}, \\
& a_{\mathrm{ef} \mathrm{II}}=\frac{\left(1.133 h+0.867 / h^{2}\right) a_{\mathrm{m} . \mathrm{v} .}}{h} .
\end{aligned}
$$

Equations (20) and (21) permit us to obtain not only the final distribution, but also the expression for any current distribution, which has formed until the arbitrary moment $\tau_{\mathrm{c}}<\tau_{\mathrm{spr}}$. For this purpose, it is sufficient to determine the values $\varphi^{*}\left(\tilde{r}_{\mathrm{fix}}, \tau_{\mathrm{c}}\right)$, $a_{\mathrm{ef}}\left(\tilde{r}_{\mathrm{fix}}, \tau_{\mathrm{c}}\right), \theta_{i}\left(\tilde{r}_{\mathrm{fix}}, \tau_{\mathrm{c}}\right), D\left(\tilde{r}_{\mathrm{fix}}, \tau_{\mathrm{c}}\right)$ at $\tau=\tau_{\mathrm{c}} \forall \tilde{r}_{\mathrm{fix}}$, which can be done by the same formulae.

\subsection{Distribution function for the theoretical laws of an ablating meteoroid}

The distribution function (Eq. (20)) was derived based on the empirical law of the meteoroid motion (Eq. (12)), which represented quantitatively well the physical phenomenon observed. To eliminate influence of the arbitrary form of the empirical law, the procedure of obtaining $f_{\mathrm{n}}(\tilde{r})$ is undertaken in a similar way, but it is grounded now on theoretical laws (Eq. (16a)). For the expressions Eq. (16a), the equation of line $\tilde{r}=\tilde{r}_{\text {fix }}$ in the $(\varphi, \tau)$-plane can be implicitly expressed as follows:

$\tau\left(\varphi ; \tilde{r}_{\mathrm{fix}}\right)=\frac{1}{(h-1) C}\left[1-\left(\frac{\tilde{r}_{\mathrm{fix}}}{B_{1} \Psi(\varphi)}\right)^{2}\right]$.

In a similar way, with the help of Eqs. (5) and (16a), we obtained the equation for the differential distribution function:

$$
\begin{aligned}
\Delta n & =f_{\mathrm{n}}\left(\tilde{r}_{\mathrm{fix}}, \tau\right) \Delta \tilde{r} \\
& =\frac{2 B_{1}^{3} B_{2}}{C(h-1) \tilde{r}_{\mathrm{fix}}^{4}} \int_{\varphi_{*}}^{\varphi^{*}}[1-C(h-1) \tau(\varphi)]^{\eta} \sin ^{2} \varphi \mathrm{d} \varphi \Delta \tilde{r},
\end{aligned}
$$

in which $\eta=3 h /(h-1)$. The variable of integration can be transformed from $\varphi$ to $2 \varphi$, so that we can use the tabular integral $\int P_{\eta}(\varphi) \cos 2 \varphi \mathrm{d} \varphi=$ $\frac{\sin 2 \varphi}{2} \sum_{k=0}^{\mathrm{E}(\eta / 2)}(-1)^{k} \frac{P_{\eta}^{(2 k)}(\varphi)}{2^{2 k}}+\frac{\cos 2 \varphi}{2} \sum_{k=1}^{\mathrm{E}((\eta+1) / 2)}(-1)^{k-1} \frac{P_{\eta}^{(2 k-1)}(\varphi)}{2^{2 k-1}} \equiv F_{\eta}(\varphi)$, which is valid for integer $\eta$ (see Gradshtein \& Ryzhyk 1980). Then, integrating along the straight $\tau-\tau_{*}=\left(\varphi-\varphi_{*}\right) / a_{\mathrm{ef}}$, we obtained the distribution function in the polynomial form

$$
f_{\mathrm{n}}\left(\tilde{r}_{\mathrm{fix}}\right)=\frac{3 h B_{1}^{3} B_{2}}{(h-1) A \tilde{r}_{\mathrm{fix}}^{4}}\left[\frac{1}{c(\eta+1)} P_{\eta+1}(\varphi)-F_{\eta}(\varphi)\right]_{\varphi_{*}}^{\varphi^{*}}
$$

Here $P_{\eta}(\varphi)=(b+c \varphi)^{\eta}$ is a polynomial, $P_{\eta}^{(k)}-$ its $k$ th derivative, $b=1-(h-1) C\left(\tau_{*}-\varphi_{*} / a_{\mathrm{ef}}\right), c=-(h-1) C / a_{\mathrm{ef}}$, and $E(x)$ is the entire part of $x$. The series of discrete values of $h$ : $1<h=\eta /(\eta-3) \leq 4$ corresponds to natural $\eta>3$. For integers $\eta<0$ we obtained a series of $h$ values, which belong to the interval $0.25 \leq h<1$ of incomplete regimes of drop fragmentation 

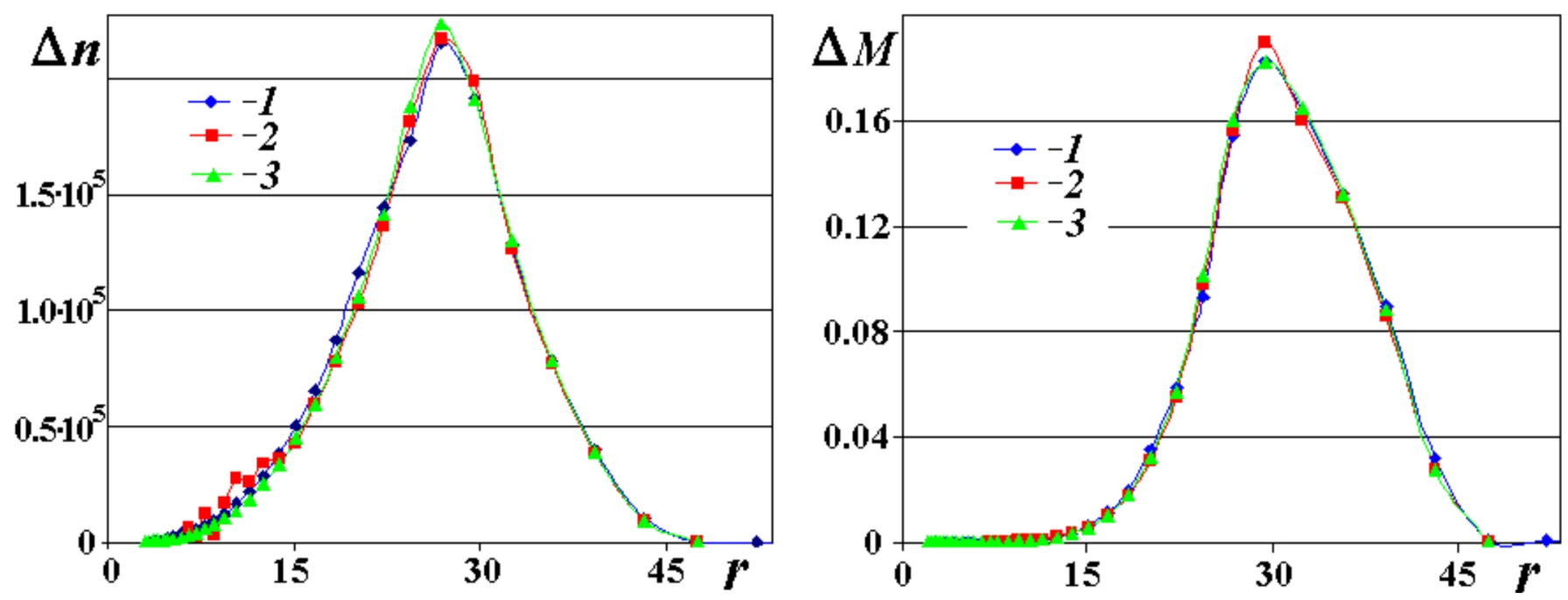

Fig. 4. Broken-away particle number, $\Delta n$, and mass, $\Delta M$, distributions; $r$ in $\mu \mathrm{m}$. Iron meteoroid, $V_{\infty}=60 \mathrm{~km} \mathrm{~s}^{-1}, \mathrm{GI}=13, \varphi_{\mathrm{lt} 0}=17.4^{\circ}, h=662$, $A=1.10, r_{\text {fix }}\left(\varphi_{\mathrm{lt} 0}\right)=26.4 \mu \mathrm{m}$. Curve 1 - calculated by Eqs. (20) and (21); curve 2 - by Eq. (25)-(27); curve 3 - by direct numerical integration of Eqs. (7) and (8) of Paper I, correspondingly.

(Girin 2011b); in this case $f_{\mathrm{n}}$ is expressed by the integral sine and cosine functions, $\operatorname{Si}(\varphi), \operatorname{Ci}(\varphi)$.

The aforementioned set of $\eta$ values covers densely enough all the practically important range of $h$. The real meteor conditions correspond to $h \gg 1$, which are consonant with $\eta=3, \eta=4$, being closer to the former value. In order to simplify the calculations, (Eq. (24)) is simplified at the upper limit, $\varphi^{*}=\pi / 2$, where $\sin 2 \varphi^{*}=0, \cos 2 \varphi^{*}=-1$ and at the lower limit for the basic range, where $\tau_{*}=0, P_{\eta}\left(\varphi_{0}\right)=1$. We give here the final formulae for the calculation of the integral in Eq. (23) for the case $\eta=3$. Namely, in sub-domain $\mathbf{I}$ :

$$
\begin{gathered}
\int_{\varphi_{*}}^{\varphi^{*}}(b+c \varphi)^{3} \sin ^{2} \varphi \mathrm{d} \varphi=\frac{1}{8 c}\left(A_{1}^{4}-1\right)+\frac{3 c}{8}\left(A_{1}^{2}-\frac{c^{2}}{2}\right) \\
+\frac{1}{4}\left(1-\frac{3}{2} c^{2}\right) \sin 2 \varphi_{0}+\frac{3 c}{8}\left(1-\frac{c^{2}}{2}\right) \cos 2 \varphi_{0},
\end{gathered}
$$

where $A_{1}=1-C(h-1) \frac{\pi / 2-\varphi_{0}}{a_{\mathrm{ef}}}$. In sub-domain II:

$$
\begin{gathered}
\int_{\varphi_{*}}^{\varphi^{*}}(b+c \varphi)^{3} \sin ^{2} \varphi \mathrm{d} \varphi=\frac{1}{8 c}\left(A_{2}^{4}-A_{3}^{4}\right)+\frac{3 c}{8}\left(A_{2}^{2}-\frac{c^{2}}{2}\right) \\
+\frac{A_{3}}{4}\left(A_{3}^{2}-\frac{3}{2} c^{2}\right) \sin 2 \varphi_{\mathrm{lt}}+\frac{3 c}{8}\left(A_{3}^{2}-\frac{c^{2}}{2}\right) \cos 2 \varphi_{\mathrm{lt}}
\end{gathered}
$$

where $A_{2}=1+c\left(a_{\mathrm{ef}} \tau_{\mathrm{lt}}+\pi / 2-\varphi_{\mathrm{lt}}\right), A_{3}=1+c a_{\mathrm{ef}} \tau_{\mathrm{lt}}$. In this case, Eq. (22) instead of Eq. (18) should be used to calculate the values $\varphi_{*}, \varphi^{*}$. The requirements of the effective slope evaluation remain the same and lead to the expressions

$$
\begin{aligned}
& a_{\text {ef I }}=\frac{\left(1.05 h+4.18 h^{-0.5}\right) a_{\text {m.v. }} a_{*}}{h a_{*}+a_{\text {m.v. }} h^{-2}+\left(a_{\text {m.v. }}+a_{*}\right) h^{-0.5}}, \\
& a_{\text {ef II }}=\frac{(1.137 h+0.867) a_{\text {m.v. }} a_{*}}{h a_{*}+a_{\text {m.v. }}} .
\end{aligned}
$$

Equations (25)-(27) permit to obtain not only the final distribution, but additionally the current distribution, which is formed to any arbitrary moment $\tau_{\mathrm{c}}<\tau_{\mathrm{spr}}$. For this purpose, it is sufficient to determine the values $\varphi^{*}\left(\tilde{r}_{\mathrm{fix}}, \tau_{\mathrm{c}}\right), a_{\mathrm{ef}}\left(\tilde{r}_{\mathrm{fix}}, \tau_{\mathrm{c}}\right)$, $\theta_{i}\left(\tilde{r}_{\text {fix }}, \tau_{\mathrm{c}}\right), D\left(\tilde{r}_{\mathrm{fix}}, \tau_{\mathrm{c}}\right)$ at the instant $\tau=\tau_{\mathrm{c}} \forall \tilde{r}_{\mathrm{fix}}$, which can be done by the same formulae.

\section{Results and discussion}

The broken-away droplet distributions calculated with the use of the empirical and theoretical meteoroid deceleration laws (Eqs. (20), (21), and (25)-(27), correspondingly) are given in Figs. 4-6 (curves 1, 2, correspondingly) for three different meteoroids, whose full input data are given in Paper I. Due to the lack of experimental data on particle size distribution in a meteor wake, the results of the present calculations are compared with those obtained by direct numerical integration of Eqs. (7) and (8) of Paper I (curves 3). One can see that the approximate formulae obtained in the present paper agree well for the high velocity meteoroids, when the assumption $\mathrm{GI} \gg \mathrm{GI}_{\mathrm{cr}}=0.4$ is fulfilled.

However, it ought to be noted that application of the Eq. (20) of the empirical law at large values of $h$ leads to numerical oscillations in the fine fraction and requires methods of higher accuracy. Nevertheless, this deviation is small when considering the mass distribution, as shown in Figs. 4-6. In contrast, Eqs. (25)-(27) based on theoretical approach, work uniformly well.

Figure 6 shows that the approximate solution is not valid if the main assumption of the presented approach, GI $\gg \mathrm{GI}_{\mathrm{cr}}$, is not satisfied. The agreement gradually becomes worse because of the influence of the "hump" in $\Delta_{\mathrm{f}}\left(W e_{\mathrm{s}}\right)$ dependence shown in Fig. 3 of Paper I, which was not taken into account in the present approximate approach. As it was noted in Paper I, this effect gives two-humped particle distribution, which cannot be reproduced by the approximate method, as shown in the example in Fig. 6 for GI $=3.55$. Hereunder, the condition $\mathrm{GI} \gg \mathrm{GI}_{\mathrm{cr}}$ establishes the limits of the elaborated approximate model application. 

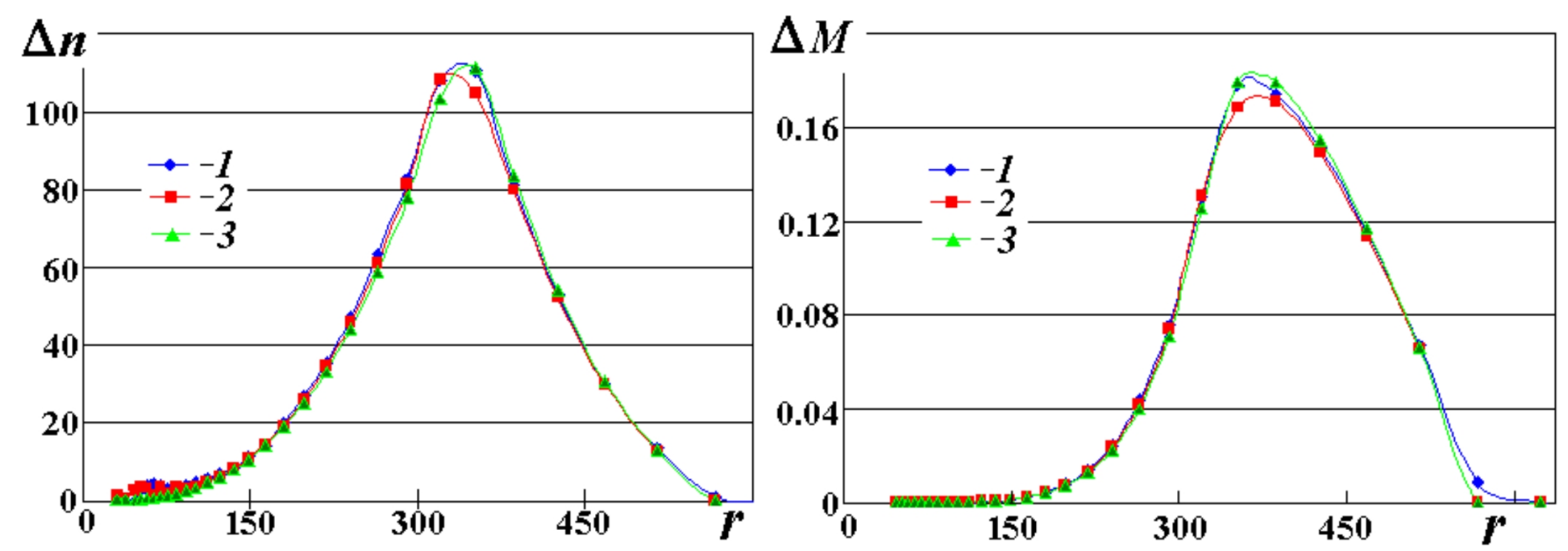

Fig. 5. Broken-away particle number, $\Delta n$, and mass, $\Delta M$, distributions; $r$ in $\mu \mathrm{m}$. Stony meteoroid, $V_{\infty}=60 \mathrm{~km} \mathrm{~s}^{-1}, \mathrm{GI}=43.5, \varphi_{\mathrm{lt} 0}=8.8^{\circ}, h=125$, $A=0.31, r_{\text {fix }}\left(\varphi_{\text {It } 0}\right)=330 \mu \mathrm{m}$. Curve 1 - calculated by Eqs. (20) and (21); curve 2 - by Eqs. (25)-(27); curve 3 - by direct numerical integration of Eqs. (7) and (8) of Paper I, correspondingly.
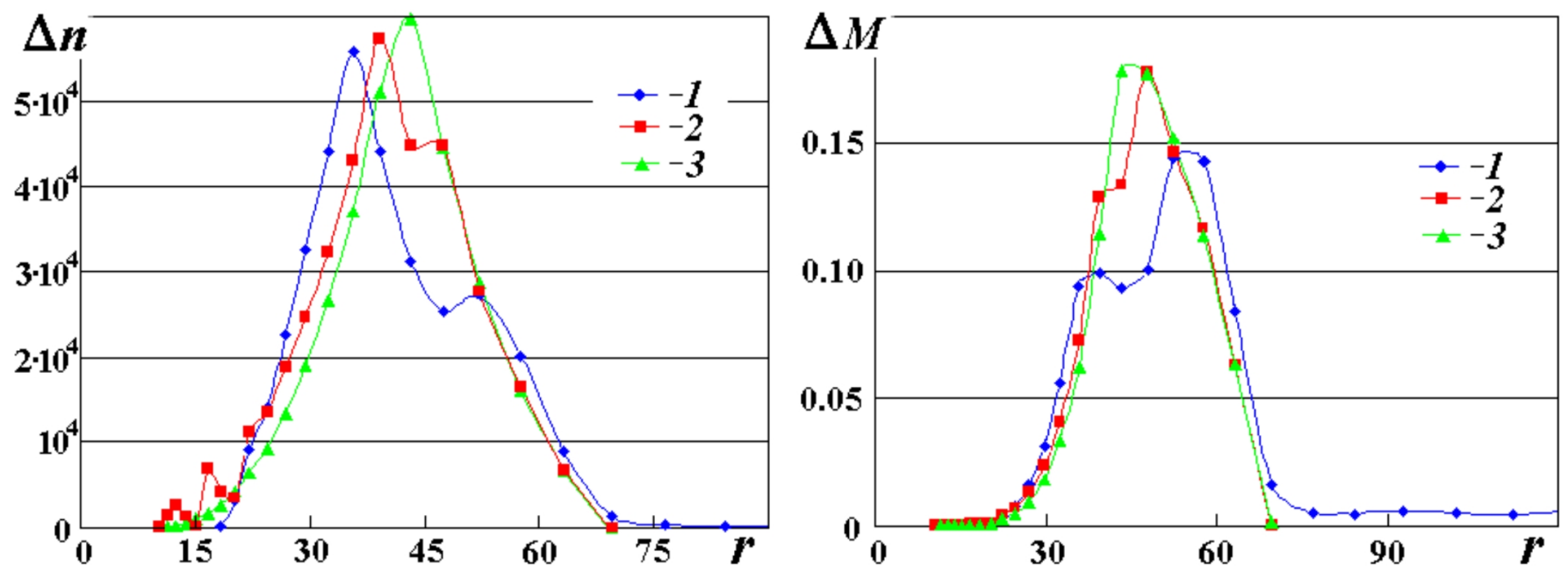

Fig. 6. Broken-away particle number, $\Delta n$, and mass, $\Delta M$, distributions; $r$ in $\mu \mathrm{m}$. Iron meteoroid, $V_{\infty}=25 \mathrm{~km} \mathrm{~s}^{-1}, \mathrm{GI}=3.55, \varphi_{\mathrm{It} 0}=31.8^{\circ}, h=662$, $A=1.10, r_{\text {fix }}\left(\varphi_{\text {It } 0}\right)=42.5 \mu \mathrm{m}$. Curve 1 - calculated by Eqs. (20) and (21); curve 2 - by Eqs. (25)-(27); curve 3 - by direct numerical integration of Eqs. (7) and (8) of Paper I, correspondingly.

\section{Conclusions}

The basic approximate relationships of the ablating meteoroid dynamics for the case of a shallow angle of the meteor trajectory are derived through the application of the theory of meteoroid melt spraying due to the mechanism of gradient instability. Three main processes, meteoroid mass loss, meteoroid deceleration and the melt droplet breakaway kinetics, are interrelated. Therefore, finding out the number distribution of the breakaway particles by sizes requires solving this trinal problem, for which approximate laws are obtained analytically in the present paper under some simplifying assumptions. The solution obtained here provides the intermediate and final number distributions in the form of the approximate relationships (Eqs. (20), (21), or (25)-(27)). Simultaneously, the meteoroid mass loss law is obtained together with the motion law of an ablating meteoroid by the integration of the governing differential equations. The verification was done by comparing the obtained approximate formulae with the solution derived through the accurate integration of the governing equations by the numerical scheme listed in Paper I and showed their agreement. The limits of the approximate solution applicability are established.

As an advantage, the theoretical approach presented herein allows analysing the ablating meteoroid dynamics. The built $(R, U)$ diagrams show that the meteoroid is always held in the regime of dynamic ablation, when the aerodynamic effect is shifted towards the spraying action, so that the majority of the airstream energy is spent to support the hydrodynamic instability of the melt, while the minor part, on the meteoroid deceleration. Thus, the meteoroid ablation proceeds at almost utmost, initial value of the relative airstream velocity. The sharp decrease of the relative velocity at the end of the meteoroid trajectory is only due to the meteoroid radius vanishing, which rises the meteoroid deceleration to infinity. Radius of the ablating meteoroid decreases near-linearly with time.

The $h$-criterion, which governs the meteoroid ablation-todeceleration rate balance, is revealed, which depends only on the air-melt density and viscosity ratios and is independent of the meteoroid velocity and size. The $h$-criterion values of an iron meteoroid are larger than those of a stony due to the smaller 
melt viscosity. Therefore, the melt spraying proceeds more efficiently for an iron than for a stony meteoroid. We remind the reader that the obtained results are valid within the frames of the main assumptions, namely, a shallow-angle meteor trajectory, the range of sizes of the completely molten meteoroids, large value of the gradient instability criterion, $\mathrm{GI} \gg \mathrm{GI}_{\mathrm{cr}}$, and meteoroid spherical shape.

The obtained relationships are necessary as the initial data for the solution of some practical problems of the meteor phenomena. Either full numerical scheme listed in Paper I, or the approximate relations obtained here, can be applied to problems of aerosol formation in meteor wakes. The presented theory of the molten meteoroid spraying can serve as the basis for elaborating future mathematical models for the aerodynamics of accelerating and evaporating mist of the broken-away melt particles, which constitutes an aerosol structure of a meteor wake, permitting to calculate the meteor light curve and to compare it with the observed one. Similar wake models were elaborated for the case of an atomizing drop in a high velocity gas flow in onedimensional (Girin 2012) and two-dimensional approximations (Girin 2014). Besides, the obtained results help us to understand the underlying dynamics of an ablating meteoroid in its connection with aerosol formation in a meteor wake, being known that they are all interrelated. The proposed theory can also be extended to a general case of a meteoroid entering atmosphere at an arbitrary entry angle.

\section{References}

Bronshten, V. A. 1983, Physics of Meteoritic Phenomena (Dordrecht: Reidel)

Drouard, A., Vernazza, P., Loehle, S., et al. 2018, A\&A 613, A54

Girin, A. G. 2011a, J. Eng. Phys. Thermophys., 84, 872

Girin, A. G. 2011b, J. Eng. Phys. Thermophys., 84, 1009

Girin, A. G. 2012, Comb. Sci. Technol., 184, 1412

Girin, A. G. 2014, At. Sprays, 24, 977

Girin, O. G. 2017, A\&A, 606, A63 (Paper I)

Gradshtein, I. S., \& Ryzhyk, I. M. 1980, Table of Integrals, Series and Products, ed. A. Jeffrey (London: Academic Press)

Madiedo, J. M., Trigo-Rodríguez, J. M., Zamorano, J., et al. 2014, A\&A, 569, A 104

Pecina, P., \& Koten, P. 2009, A\&A, 499, 313

Ranger, A. A. 1972, Astron. Acta, 17, 675

Reinecke, W. G., \& Waldman, G. D. 1975, Shock Layer Shattering of Cloud Drops in Reentry Flight (AIAA Paper, No. 152, 22 pp.)

Simonenko, A. N. 1974, Astronomicheskiy Vestnik (Astronomic Herald), 8, 165 (in Russian)

\section{Appendix A: Nomenclature}

$B_{1}, B_{2}$

$\mathrm{GI} \equiv W e_{\infty} R e_{\infty}^{-0.5}$

$h=A / 3 C$

$\operatorname{Im}^{-1}(\omega)$

$k_{\mathrm{r}}, k_{\mathrm{t}}$

$K, L, Q$

$m$

$M \equiv m / m_{0}$

$R$

$\tilde{R} \equiv R / R_{0}$

$r$

$\tilde{r} \equiv r / R_{0}$

$R e_{\infty} \equiv \rho_{\infty} 2 R_{0} V_{\infty} / \mu_{\infty}$ scaling parameters

gradient instability criterion

rate ratio of ablation-to-

deceleration processes

growth rate factor

coefficients in Eq. (3)

constants

meteoroid mass

current meteoroid radius

breakaway droplet radius

Reynolds number for meteoroid $t$

$t_{\mathrm{ch}} \equiv 2 R_{0} / \alpha^{0.5} V_{\infty}$

V

$w$

$W \equiv w / V_{\infty}$

$W e_{\infty} \equiv \rho_{\infty} V_{\infty}^{2} 2 R_{0} / \Sigma \quad$ Weber number for meteoroid

$W e_{\mathrm{s}} \equiv \rho_{\mathrm{m}} V_{\mathrm{s}}^{2} \delta_{\mathrm{m}} / \Sigma$

$x_{\mathrm{M}}$

$X_{\mathrm{M}}=x_{\mathrm{M}} / 2 R_{0}$

$z$

elapsed time

characteristic timescale

velocity

meteoroid velocity deficit due to aerodynamic drag

surface Weber number

meteoroid displacement due to

aerodynamic drag

meteor altitude.

\section{Greek symbols:}

$\alpha \equiv \rho_{\mathrm{a}} / \rho_{\mathrm{m}}$

$\gamma$

$\Gamma$

$\delta$

$\mu_{\mathrm{a}, \mathrm{m}}$

$\mu \equiv \mu_{\mathrm{a}} / \mu_{\mathrm{m}}$

$\rho$

$\Delta l$

$\Sigma$

$\tau=t / t_{\mathrm{ch}}$

$\lambda$

$\varphi$

$\Delta n$

$\Delta \equiv 2 \pi \delta_{\mathrm{m}} / \lambda$

$\Delta v_{\text {tor }}$

$\tau_{\text {ind }}$

$\omega$

$\Omega \equiv \omega \delta_{\mathrm{m}} / V_{\mathrm{s}}$

air-to-melt density ratio

angle between trajectory and horizon

aerodynamic drag coefficient

boundary layer thickness

dynamic viscosity

air-to-melt viscosity ratio

density

area element length

melt surface tension

dimensionless time

disturbance wavelength

polar angle of the surface

area element

number of newborn droplets

dimensionless wavenumber

torus volume

disturbance induction time

disturbance complex frequency

Indices:

' $\mathrm{a}^{\prime} \quad$ air near meteoroid surface

' $\mathrm{c}^{\prime} \quad$ current

'cr' critical

'ef' effective

' $\mathrm{f}$ ' fastest disturbance

'fix' fixed

'ind' induction

'ir' iron

' $1 t^{\prime} \quad$ left boundary

'm.v.' mean value

'm' melt

' $\mathrm{M}^{\prime} \quad$ meteoroid

' $n$ ' number

'per' period

' $\mathrm{r}$ ' radius

' $\mathrm{rt}^{\prime}$ right

's' surface

'st' stone

'spr' spraying

' $\mathrm{t}$ ' time

${ }^{\prime} 0^{\prime} \quad$ initial values

$' \infty$ ambient air 